\title{
Differences between NSA 94-106 and IEEE 299 LF magnetic shielding measurements
}

\author{
Johan Catrysse*, Filip Vanhee, Davy Pissoort \\ ReMI, Reliability in Mechatronics and ICT \\ KU Leuven, Technology Campus Oostende, \\ Zeedijk 101, B 8400 Oostende (Belgium) \\ *MICAS, ESAT, KU Leuven (Leuven)
}

\begin{abstract}
For the characterisation of the LF magnetic shielding of conductive materials two setups are available. Although for both methods, loop antennas are used, two different configurations are defined. The one referenced in NSA 94-106 (formerly NSA 65-6) is called the parallel setup. The one referenced in IEEE 299 (formerly MIL STD 285) is called the coplanar setup. When performing measurements under similar conditions, different values for the resulting shielding effectiveness (SE) are obtained. This paper will discuss the background why differences are occurring.
\end{abstract}

Keywords: LF magnetic shielding, NSA 94-206, IEEE 299

\section{INTRODUCTION}

For the characterization of the LF magnetic shielding on conductive materials two setups are available. Both are based on the use of loop antennas, but the physical setup is different.

One is referenced in NSA 94-106 (formerly NSA 65-6) [1] and is known as the parallel setup, where both transmitting and receiving antennas are in parallel planes, and parallel with the plane of the sample under test. This is sketched in figure1.

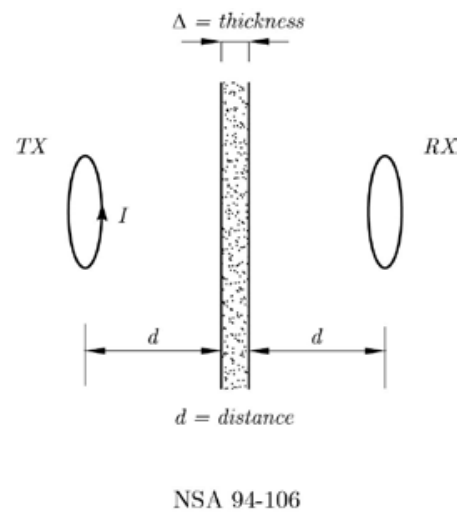

Figure 1. Basic setup for NSA 94-106 LF magnetic SE measurement

The other one is referenced in IEEE Std 299 (formerly also MIL STD 285) [2] and is known as the coplanar setup, where both antennas are located in the same plane, but perpendicular to the sample. This is sketched in figure 2.

When performing SE measurements using both methodologies, but identical samples and equipment, different results are obtained for the resulting SE values.

\author{
Salvatore Celozzi \\ Electrical Engineering Division - DIAEE \\ University of Roma "La Sapienza" \\ via Eudossiana 18, IT 00184 Roma (Italy)
}

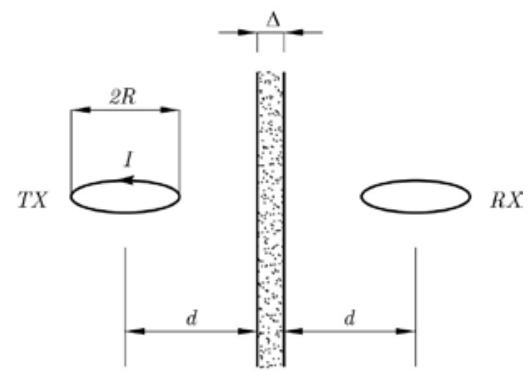

MIL-STD 285

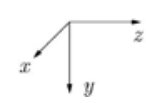

Figure 2. Basic setup for IEEE Std 299 LF magnetic SE measurement

Measurements are performed using a metal cage with an open window in one wall. The measurement setup is sketched in figure 3. A more detailed view of the fixture in order to clamp the samples is shown in the figures 4 .

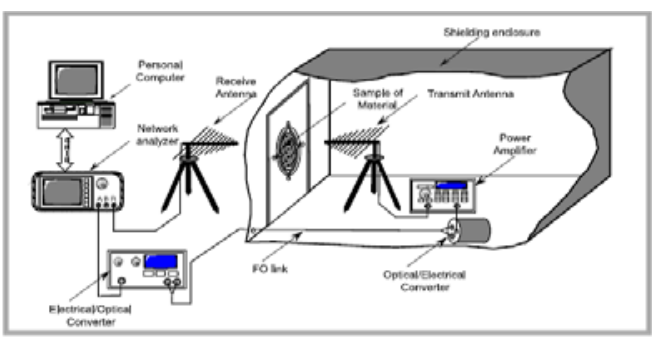

Figure 3. Global sketch of the measuring setup

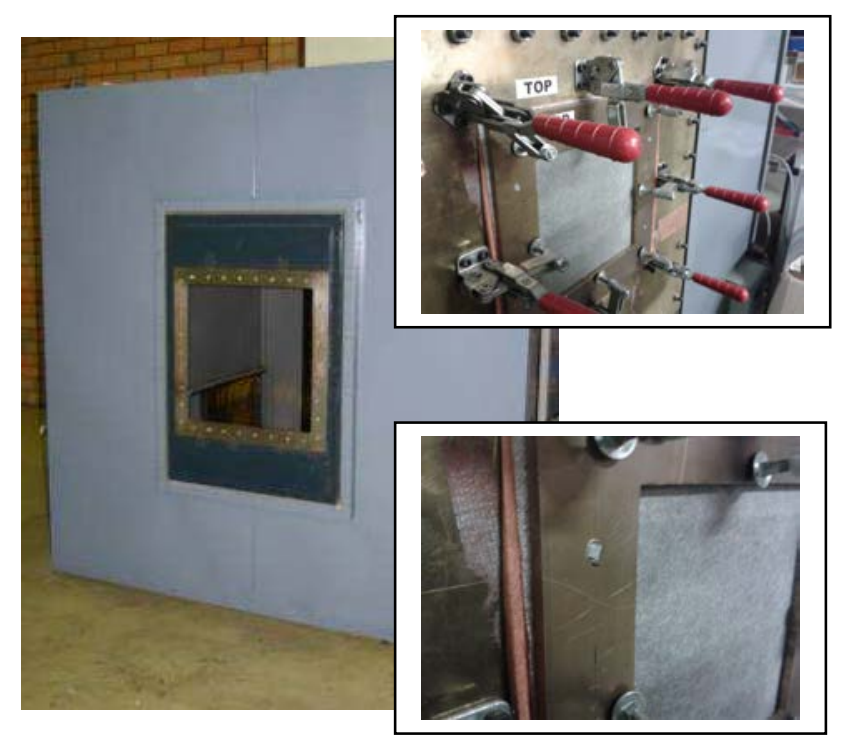




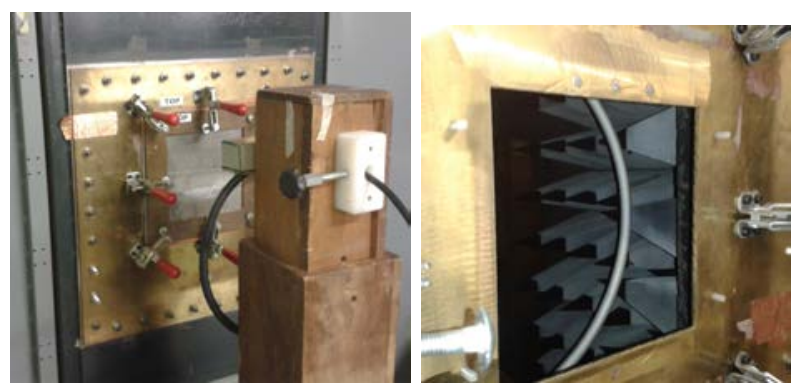

Figure 4. Pictures of the measuring setup

\section{SOME THEORETICAL BACKGROUND}

Theoretical analysis has been done in the past by different authors, as Bannister [9]-[10], Dahlberg and others. This resulted in some general integral formulations of the SE values, and also in some closed form approximations under certain conditions.

Recently, Celozzi [3] made an interesting analysis of both setups and the most important results of this work are summarized in the next paragraphs.

\subsection{NSA 94-106 setup}

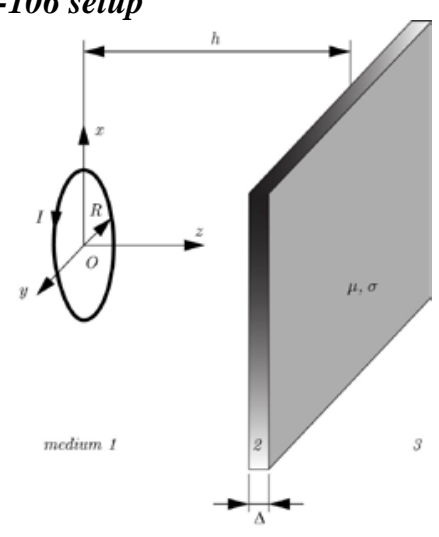

Figure 5. NSA 94-106 parallel setup

The exact solution for the SE, accounting for the physical dimensions of the transmitting loop antenna and considering as observation point the symmetrical one beyond the shield, is given by formula (1) [3]. For both antennas, the centre point is considered as reference and the distance between both is $r$.

$$
\begin{aligned}
& S E=\frac{1}{4 \mu_{r}} \frac{\int_{0}^{\infty} \frac{\lambda^{2}}{\tau_{0}} J_{1}(\lambda R) J_{0}(\lambda r) e^{-\tau_{0} z} d \lambda}{\frac{\lambda^{2} T}{\tau_{0}^{2}} J_{1}(\lambda R) J_{0}(\lambda r) e^{-\tau_{0} z-\left(r-\tau_{0}\right) \Lambda_{d}} d \lambda} \\
& K=\left[\left(\frac{\tau}{\tau_{0}}+\mu_{r}\right)^{2}-\left(\frac{T}{\tau_{0}}-\mu_{r}\right)^{2} e^{-2 r \Lambda}\right]^{-1} \\
& \tau_{0}=\sqrt{\lambda^{2}-k_{0}^{2}} \\
& \tau=\sqrt{\lambda^{2}-\gamma^{2}} \\
& k_{0}=\omega / c_{0} \\
& \gamma \cong \sqrt{j \omega \mu_{0} \mu_{r} \sigma}
\end{aligned}
$$

Important in this configuration is the dependency of the resulting SE from only the total distance $r$ between both antennas, the radius $\mathrm{R}$ of the transmitting antenna and the thickness $\Delta$ of the sample.

When translating this configuration into a Transmission Line (TL) equivalent, it requires to consider the following correct wave impedance, related to a small dipole source parallel to an conductive plane [5].

$$
\eta_{w}=\eta_{0} \frac{\left(\frac{j \omega z}{c}\right)^{2}+\left(\frac{j \omega z}{c}\right)}{\left(\frac{j \omega z}{c}\right)^{2}+3\left(\frac{j \omega z}{c}\right)+3}
$$

where $\mathrm{z}$ is the distance from the observation point and $\mathrm{c}$ the speed of light in free space. In order to apply the TL equivalent, $\mathrm{z}=\mathrm{h}$ at the position of the shield plane.

Although both the tangential electric and magnetic field components tend to vanish as the observation point tend to the loop axis, their ratio i.e. the wave impedance, is constant. Its LF approximation is valid when

$$
\frac{\omega z}{c} \ll \mathbf{1}
$$

which implies, considering a loop radius $\mathrm{R}$ and its distance from the shield as requested by IEEE Std. 299 $(2 \mathrm{R}=\mathrm{z}=305 \mathrm{~mm})$, a maximum frequency of $16 \mathrm{MHz}$. As further tests were performed up to $20 \mathrm{MHz}$, the small dipole LF approximation may readily be used:

$$
\eta_{w}=\eta_{0}\left(\frac{j \omega z}{3 c}\right)=j \omega \mu_{0} \frac{z}{3}
$$

Regarding a practical measurement setup, the source will have some finite dimensions. Therefore, a correction, ensuing from the exact integral expression, may be introduced to account for these finite dimensions of the transmitting source [3], [12]:

$$
\eta_{w}=j \omega \mu_{0} \frac{\left(R^{2}+z^{2}\right)}{3 z}
$$

This shows a very strong dependency of the wave impedance with respect to the radius $\mathrm{R}$ of the transmitting antenna. It means that, even when maintaining all distances from the antennas to the sample, other values for SE might be obtained when changing the radius $\mathrm{R}$ of the transmitting antenna.

\subsection{IEEE Std 299 setup}

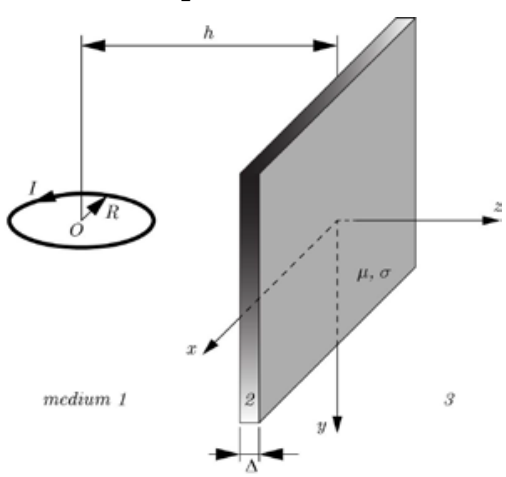

Figure 6. IEEE Std 299 coplanar setup 


$$
\begin{aligned}
S E & =\frac{\int_{0}^{\infty} \int_{0}^{\infty} \frac{\beta I_{1}(\beta R)}{D_{1}} \cos (\alpha x) \cos (\beta y) e^{-D_{1}|-\hbar|} d \alpha d \beta}{\int_{0}^{\infty} \int_{0}^{\infty} \frac{4 \beta I_{1}(\beta R) D_{3} e^{-\left(D_{2}-D_{1} \mid \Lambda\right.}}{D_{4}} \cos (\alpha x) \cos (\beta y) e^{-D_{1} \mid z-\phi} d \alpha d \beta} \\
D_{1} & =\sqrt{\alpha^{2}+\beta^{2}} \\
D_{2} & =\sqrt{\alpha^{2}+\beta^{2}+j \omega \mu \sigma} \\
D_{3} & =D_{2} / \mu_{r} \\
D_{4} & =\left(D_{3}+D_{1}\right)^{2}-\left(D_{3}-D_{1}\right)^{2} e^{-2 D_{3} \Delta}
\end{aligned}
$$

From the formulas (6) [3], [5]-[7], the direct dependency of the resulting SE from the distance $h$ (from the transmitting antenna to the sample), the radius $\mathrm{R}$ of the transmitting antenna and the thickness $\Delta$ of the sample is observed for this configuration.

Celozzi reports also on the resulting SE by using exact formula's and different approximations for a set of materials [3], and by assuming an infinite planar shield. In the NSA 94-106 configuration, tangential components are only generated in points away from the loop axis. The larger the region that may be taken into account, the larger is the observed effect in the field distribution, and consequently in the related SE value.

In the IEEE Std. 299 configuration, tangential components are exactly those who propagate through the shield, according to related TL simulation models. The difference of the dimensions of the transmitting loop antenna is not so relevant when the radius $\mathrm{R}$ is changed (under the condition of maintaining an identical current) [6] - [7].

It follows that different SE values may be obtained by using one of the two measuring setups.

Under conditions of $\mathrm{R}=15.25 \mathrm{~cm}$ and the distance $\mathrm{h}$ from both antennas to the sample $=30.5 \mathrm{~cm}$, it turns out that NSA 94-106 SE values tend to be some $\mathrm{dB}$ lower than the IEEE Std 299 ones.

More details can be found in appendix B of [3].

\section{MEASUREMENTS}

Measurements have been performed on a number of different samples of materials, and 2 typical ones are summarised:

- Aluminium foil, thickness $0.1 \mathrm{~mm}$

- Calandered foil, stainless steel fibers, thickness $1 \mathrm{~mm}$

Measurements are performed using a metal cage of $2 \times 2 \times 2 \mathrm{~m}^{3}$, with an open window in one wall of $50 \times 50$ $\mathrm{cm}^{2}$. The receiving antenna is a battery operated loop antenna EMCO 6507 and the transmitting antennas are "home made", so that they can handle up to 10 Watt transmitting power.

There is a set of three antennas with a different radii (32 $\mathrm{cm}, 20 \mathrm{~cm}$ and $7 \mathrm{~cm}$ respectively), due to the selfresonance of the antennas, and they cover the frequency range in three frequency-bands from $20 \mathrm{KHz}-200 \mathrm{KHz}$ - $2 \mathrm{MHz}-20 \mathrm{MHz}$.
The resulting SE values for the NSA 94-106 setup are each time dropping when changing the transmitting antenna at the frequencies of $200 \mathrm{KHz}$ and $2 \mathrm{MHz}$ (blue lines in figures 7 and 8).

As it is assumed that the SE should not show a discontinuity at the same frequency, graphs are corrected with the difference between both SE values at these frequencies, in order to assume a continuous behaviour of the SE (red lines in figures 6 and 7).

So, the next graphs are showing three curves:

- NSA 94-106, as measured

- NSA 94-106 corrected for changing antenna radius R - IEEE Std 299, as measured
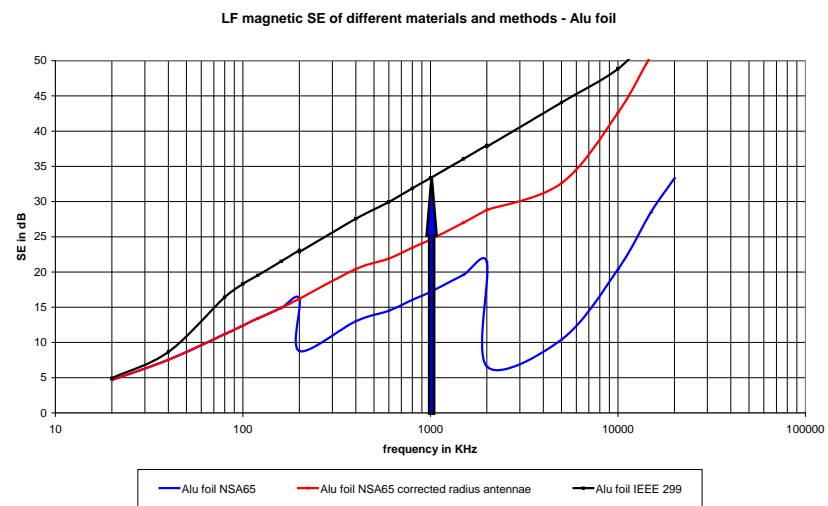

Figure 7. SE of the Aluminium foil

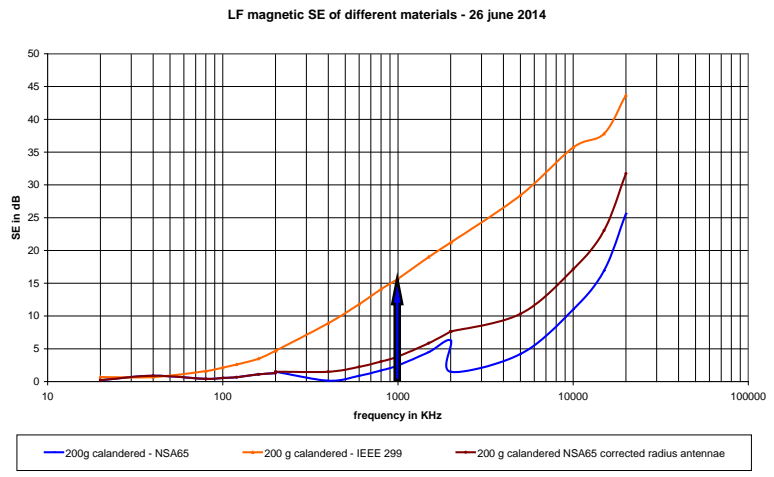

Figure 8. SE of 1 layer of $200 \mathrm{~g}$ calandered stainless steel foil

\section{WAVE THEORY}

Schelkunoff published in 1938 a paper on the concept of using impedance in order to simulate shielding problems [4]. It is known as the wave theory applied to shielding problems of the transmission line model. As a result, the following table summarises formula's for the estimation of SE under different conditions.

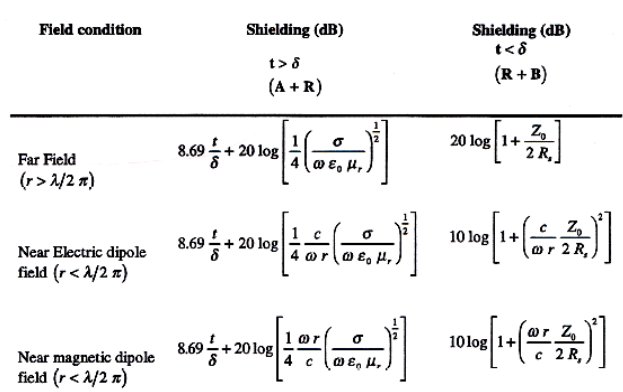


Under the conditions of near field and a thin material, the magnetic field SE is given by the formula (token from the previous table):

$$
10 \log \left[1+\left(\frac{\omega r}{c} \frac{Z_{0}}{2 R_{s}}\right)^{2}\right]
$$

with $\mathrm{R}_{\mathrm{s}}$ the DC square resistance of the material $=1 /(\sigma . \mathrm{t})$

The DC square resistance of both samples has been measured:

\begin{tabular}{|l|l|}
\hline \multicolumn{1}{|c|}{ sample } & $\begin{array}{c}\text { DC resistance } \\
\text { (sqohm) }\end{array}$ \\
\hline Aluminium foil & 0.058 \\
\hline Calandered SS foil & 0.228 \\
\hline
\end{tabular}

In 1969, a paper was published by A. Whitehouse [12] calculating exact wave impedances for both the parallel loop and the coplanar loop cases, and where it turned out that the LF magnetic near field approximation made by Schelkunoff is only correct for the coplanar loop or IEEE Std 299 configuration.

In case of parallel loops or the NSA 94-106 configuration, the following LF approximation can be made for the $\mathrm{SE}=\mathrm{R}+\mathrm{B}$ [11] in case of thin samples:

$$
\begin{aligned}
& R \cong-20 \log _{10}\left|\frac{4 \eta}{\eta_{w}+2 \eta}\right| \\
& B=20 \log _{10}\left|\frac{\left(\eta_{w}+\eta\right)^{2}-\left(\eta_{w}-\eta\right)^{2} e^{-2 \gamma t}}{\left(\eta_{w}+\eta\right)^{2}}\right| \simeq \\
& 20 \log _{10}\left|\frac{\eta_{w} 2 \gamma t+4 \eta}{\eta_{w}+2 \eta}\right| \\
& \text { where } \gamma=\sqrt{j \omega \mu \sigma} .
\end{aligned}
$$

Finally, the approximated SE is obtained as:

$$
\mathrm{SE}=20 \log _{10}\left|\frac{\eta_{w \sigma} 2 \gamma t+4 \eta}{4 \eta}\right|=20 \log _{10}\left|1+j \omega \mu_{0} \frac{z}{3} \frac{\sigma t}{2}\right|
$$

Comparing this NSA 94-106 approximation with the Schelkunoff formula, a difference by a factor of $1 / 3$ is observed, or a difference in SE of $10 \mathrm{~dB}$ may be expected in practice.

Based on the measured DC square resistances, and the thickness of the materials, the conductivity $\sigma$ may be obtained. Using the above estimations of the LF magnetic field SE, as defined by Whitehouse and by Schelkunoff, the calculated SE at $1 \mathrm{MHz}$ at a distance of $30 \mathrm{~cm}$ between source and sample is given in the next table:

\begin{tabular}{|l|l|l|}
\hline \multicolumn{1}{|c|}{ sample } & \multicolumn{2}{c|}{ LF Magnetic SE in dB } \\
\hline & \multicolumn{1}{|c|}{ Whitehouse } & Schelkunoff \\
\hline Aluminium foil & $25.98 \mathrm{~dB}$ & $35.88 \mathrm{~dB}$ \\
\hline $\begin{array}{l}\text { Calandered } \\
\text { stainless steel foil }\end{array}$ & $4.9 \mathrm{~dB}$ & $14.5 \mathrm{~dB}$ \\
\hline
\end{tabular}

Comparing these calculated SE values with the measured ones as given in the figures 6 and 7, a good agreement is obtained with respect to both NSA 94-106 and IEEE Std 299 methods.

\section{CONCLUSIONS}

In this paper, the differences in LF magnetic field SE between the NSA 94-106 and the IEEE Std 299 methods has been described from the practical point of view.

Measurements show a difference in SE values, and concerning the NSA 94-106 method, the direct impact of the radius of the transmitting antenna has been demonstrated.

Resulting SE values obtained by the IEEE Std 299 are in good agreement with the theoretical wave model as defined by Schelkunoff.

\section{REFERENCES}

[1] NSA 94-106, National Security Agency, Specification for shielded enclosures, Fort George Meade, MD,1994

[2] IEEE Std 299'T - 2006, IEEE Standard method for measuring the shielding effectiveness of electromagnetic shielding enclosures, Piscataway, NJ, 2007

[3] S; Celozzi, et al., Electromagnetic Shielding, IEEE Press/J.Wiley, 2008

[4] Schelkunoff, The impedance concept and its application to problems of reflection, refraction, shielding and power absorption, Bell System Technical Journal, BSTJ 17-1, pp. 17-48, Jan. 1938

[5] S. Celozzi and M. D'Amore, "Magnetic field attenuation of nonlinear shields", IEEE Trans. on EMC, Aug. 1996, Vol. 38, 3, pp. 318-326

[6] S. Celozzi, "Shielding Effectiveness Prediction in MIL STD 285 Loop Source Configuration", Proc. IEEE Int. EMC Symposium 1998, Denver, Aug. 1998, pp. 1125-1130

[7] R.Araneo and S. Celozzi, "Exact solution of the low-frequency coplanar loops shielding configuration", IEE Proc. Sci. Meas. Technology, Vol. 140, No. 1 , January 2002

[8] J.R.Moser, "Low Frequency shielding of a circular loop electromagnetic field source", IEEE Trans. on EMC, Vol. 9, March 1967, pp. 6-18

[9] P. Bannister, "New Theoretical Expressions for Predicting Shielding Effectiveness for the Plane Shield Case", IEEE Trans. on EMC, Vol. 10, 1, March 1968

[10] P. Bannister, "Further Notes for Predicting Shielding Effectiveness for the Plane Shield Case", IEEE Trans. on EMC, Vol.11, 2, May 1969

[11] R.B. Schulz et al., "Shielding Theory and practice", IEEE Trans. on EMC, Vol. 30,3, Aug. 1988

[12] A. Whitehouse, "Screening: new wave impedance for the transmission line analogy", IEE Proc., Vol.116, No.7, July 1969, pp.1159-1164 